\title{
Growth rate of small pancreatic neuroendocrine tumors in multiple endocrine neoplasia type 1: results from an endoscopic ultrasound based cohort study
}

\author{
Authors \\ Wouter F. W. Kappelle ${ }^{1}$, Gerlof D. Valk², Max Leenders ${ }^{1}$, Leon M. G. \\ Moons ${ }^{1}$, Auke Bogte ${ }^{1}$, Peter D. Siersema ${ }^{1}$, Frank P. Vleggaar ${ }^{1}$ \\ Institutions \\ 1 Department of Gastroenterology and Hepatology, University \\ Medical Center Utrecht, Utrecht, The Netherlands \\ 2 Department of Endocrine Oncology, University Medical Center \\ Utrecht, Utrecht, The Netherlands
}

submitted 22.6.2016

accepted after revision 26.9.2016

Bibliography

DOI http://dx.doi.org/10.1055/s-0042-119402

Published online: 14.12.2016 | Endoscopy 2017; 49: 27-34

(c) Georg Thieme Verlag KG Stuttgart · New York

ISSN 0013-726X

\section{Corresponding author}

Wouter F. W. Kappelle, MD, Department of Gastroenterology and Hepatology, University Medical Center Utrecht, P.O. Box 85500 , 3508 GA Utrecht, The Netherlands,

Fax: +31-88-7555533,

w.f.w.kappelle@umcutrecht.nl

\section{ABSTRACT}

Background and aims In multiple endocrine neoplasia type 1 (MEN1), endoscopic ultrasound (EUS) is used for identification and follow-up of pancreatic neuroendocrine tumors (PNETs). The role of EUS in surveillance of small ( $<20 \mathrm{~mm}$ ) PNETs is unclear, mostly because the natural course of these lesions is largely unknown. We aimed to determine annual growth and incidence rate of small PNETs in patients with MEN1 using EUS-based surveillance.

Patients and methods Linear array EUS procedures in patients with MEN1 between 2002 and 2015 were identified. Number, size, and location of PNETs were recorded. Annual growth of PNETs $<20$ $\mathrm{mm}$ identified at the initial EUS ("prevalent" PNETs) and during follow-up ("incident” PNETs) was calculated using mixed model linear regression analysis.

Results A total of 54 patients were identified and 38 patients were included. In all, 226 PNETs were identified (median size $5.0 \mathrm{~mm}$, interquartile range $3.7-7.5$ ) of which 124 (55\%) were prevalent and 102 (45\%) were incident PNETs. Annual incidence rate was 0.79 PNETs/year (95\% confidence interval $[\mathrm{Cl}] 0.73$ to 0.87 ). Overall growth rate was $0.10 \mathrm{~mm} /$ year $(95 \% \mathrm{Cl} 0.02$ to $0.19 ; P=0.01)$; PNETs $<10 \mathrm{~mm}(\mathrm{n}=198)$ did not grow $(P=0.23)$, whereas PNETs $\geq 10 \mathrm{~mm}$ $(\mathrm{n}=28)$ grew $0.44 \mathrm{~mm} /$ year $(95 \% \mathrm{Cl} 0.10$ to $0.78 ; P=0.01)$. Prevalent PNETs grew $0.21 \mathrm{~mm} /$ year $(95 \% \mathrm{Cl} 0.10-0.32 ; P<0.001)$, whereas incident PNETs did not grow $(P=0.26)$.

Conclusions The annual growth rate of small, solid PNETs in patients with MEN1 is lower than previously thought. Surveillance intervals could probably be prolonged without compromising safety.

\section{Introduction}

Multiple endocrine neoplasia type 1 (MEN1) is a rare genetic disorder (prevalence 3:100 000) with autosomal-dominant inheritance [1]. A mutation in the MEN1 gene on chromosome $11 q 13$, a tumor suppressor gene, leads to hyperplastic and neoplastic disorders of endocrine organs, including the parathyroid gland (95\%), anterior pituitary gland (30\%), and pancreas [2-4]. Pancreatic neuroendocrine tumors (PNETs) are found in $30 \%-75 \%$ of patients with MEN1 and are most often nonfunctional $[5,6]$. Nevertheless, the presence of nonfunctional PNETs is associated with an increased risk of MEN1-related premature death [2,7]. An early diagnosis and timely intervention is thought to lead to a better long-term outcome [8]. Therefore, screening and surveillance of PNETs in MEN1 is warranted, even if the patient is asymptomatic.

Endoscopic ultrasound (EUS) is the most sensitive modality to screen for PNETs and enables visualization of small lesions that are frequently missed with computed tomography (CT), magnetic resonance imaging (MRI), or somatostatin receptor scintigraphy $(P<0.001)[4,9,10]$. Functional PNETs are often surgically removed to treat the endocrine syndrome (independent of size), especially when medical therapy fails [11]. There is consensus that nonfunctional PNETs larger than $20 \mathrm{~mm}$ should be surgically removed because of the increased risk of metastasis. A conservative approach is considered to be safe for smaller nonfunctional PNETs [11-15]. Nevertheless, some guidelines advise to consider surgical resection of nonfunctioning PNETs $>10 \mathrm{~mm}$ because of a scarcity of evidence-based data on the risk of metastasis of PNETs of $10-20 \mathrm{~mm}$ [11]. Furthermore, surgical resection is suggested for PNETs that show a significant growth rate (e.g. $\geq 5 \mathrm{~mm}$ per year) and exceed 10 $\mathrm{mm}$ in size. However, because of the potential morbidity of surgery, this approach requires an informed patient choice, ideally based on scientific evidence. Currently, little is known about the growth rate of small PNETs and the proportion of small PNETs that progress to large PNETs. Furthermore, little is known about annual incidence rates of new PNETs. Therefore, the aims of this study were to evaluate the growth rate and annual incidence of small PNETs (<20 mm) in patients with MEN1 using EUS in a large cohort of patients. 


\section{Patients and methods}

\section{Patients}

This study was conducted at the Department of Gastroenterology and Hepatology of the University Medical Center Utrecht, The Netherlands-a tertiary referral center for patients with MEN1. The research protocol was approved by the institutional review board.

Patients were identified using a prospective database of MEN1 patients who were under endoscopic follow-up, using the key words "EUS" and "MEN1." Procedures between May 2002 and April 2015 were included. Patients were eligible for inclusion if they were aged $\geq 18$ years and had undergone at least two EUS procedures for evaluation of the pancreas for the presence of NETs. The diagnosis of MEN1 was based either on genetic analysis or on commonly used and accepted clinical criteria, stating that at least one MEN1 manifestation combined with at least one first-degree family member with genetically proven MEN1 is required for a diagnosis [11].

Demographic data and data on (previous) surgical treatment of PNETs were recorded. Furthermore, reports of CT/MRI of the pancreas less than 1 year before surgery were reviewed.

\section{Endoscopic ultrasound procedures}

In accordance with guidelines, patients with MEN1 underwent surveillance imaging annually [11]. In our center, surveillance imaging of the pancreas is done with CT or MRI alone in the majority of patients. EUS is used to monitor multiple small PNETs, when there is doubt about growth of small lesions on CT/MRI, or when symptomatology suggests a functional NET without a visible lesion on CT/MRI.

Procedures were performed by four experienced ultrasonographers (>150 annual EUS procedures), who utilized the same technique of EUS surveillance [16]. The senior author of the paper (F.P.V.) performed the majority of procedures (78\%). Procedures were performed using a linear array echoendoscope (GF-UC140P, GF-UCT140-AL5, or GF-UCT180; Olympus, Hamburg, Germany). Patients were placed in the left lateral decubitus position and vital functions were monitored during the procedure. Conscious sedation using intravenous midazolam and fentanyl was administered in the majority of the procedures (96\%); the remaining procedures were performed with monitored anesthesia care using intravenous propofol and alfentanil.

Procedural data were collected, including ultrasonographer, date of procedure, and echoendoscope used. Data on PNETs included number, size, location, and appearance. Although EUS appearance of PNETs may vary, a typical PNET is solid, with or without a cystic component, well demarcated, hypoechoic, and homogeneous ( $>$ Fig. 1) [4]. All pancreatic lesions meeting these criteria were considered to be PNETs, which is standard practice in EUS surveillance of PNETs in patients with MEN1 [4, 17 - 19]. In the majority of (asymptomatic) patients with MEN1, one or multiple PNETs are found and for this reason, histology of detected pancreatic lesions is not regularly obtained [2023]. Lesions described as cysts, without a halo sign or a solid component, were considered to be pancreatic cysts or side-

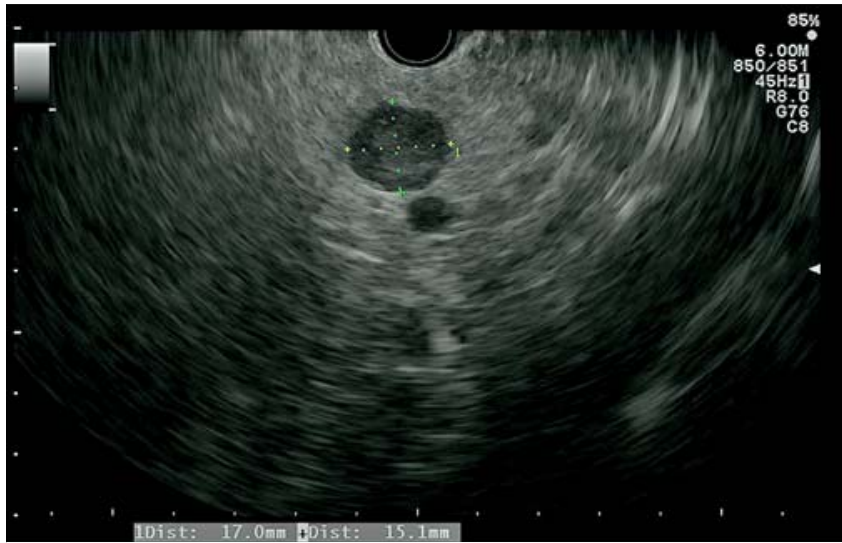

Fig. 1 Typical round, hypoechogenic, well-demarcated appearance of a pancreatic neuroendocrine tumor.

branch intraductal papillary mucinous neoplasms and were excluded from the analyses.

PNETs $\geq 20 \mathrm{~mm}$ during the initial EUS were excluded from the growth rate analysis. Only PNETs that were identified again during at least one follow-up EUS were considered for analysis, the exception being new PNETs visualized during the last EUS procedure [17]. PNET measurements were grouped with previous measurements based on the reported location in the pancreas (e. g. measurements of PNETs in the pancreatic head were grouped with previous measurements of PNETs in the pancreatic head). If multiple PNETs were found in one region, measurements were allocated based on the description in the endoscopy report (e.g. appearance or proximity to certain landmarks of the PNET). If such a description was not available, allocation was based on size; the largest lesion within a region during the previous EUS was assumed to be the largest lesion during the next EUS.

Distinction was made between PNETs that were visualized during the first EUS procedure ("prevalent" PNETs) and PNETs visualized during a subsequent EUS procedure ("incident" PNETs). For a subgroup analysis, PNETs were categorized by diameter of the longest axis at first visualization into five classes: (1) 0-4mm, (2) $5-9 \mathrm{~mm}$, (3) $10-14 \mathrm{~mm}$, (4) $15-19 \mathrm{~mm}$, (5) $\geq 20 \mathrm{~mm}$ [24].

\section{Study outcomes}

The primary outcomes of the study were the growth rate of small (<20 mm) PNETs, measured as tumor diameter of the longest axis, and incidence of new PNETs that were not identified during previous EUS.Secondary outcomes were number of PNETs that increased $\geq 2$ categories (at least $5 \mathrm{~mm}$ ) in size during follow-up, and the proportion of surgically removed PNETs that were also detected with CT or MRI.

\section{Statistical analysis}

Categorical variables were expressed as proportions. Continuous variables were expressed as median (interquartile range $[I Q R])$ or mean (SD), where appropriate. The growth rate of each lesion was calculated with linear mixed model analysis 
with random intercepts per patient and lesion to adjust for multiple observations per lesion in the same patient. Annual incidence of new PNETs was calculated by dividing the number of new PNETs by the total follow-up time (i.e. time between first and last procedure) of all patients combined. If a previously detected lesion was not described during a follow-up EUS, but was visualized during an EUS thereafter, the missing measurement was not considered in the analysis. Growth rates were compared by adding a multiplicative interaction term to the model and examining the significance.

Analyses were performed using R, version 3.3.0 (R Development Core Team 2016) or SPSS version 23 (IBM Corp., Armonk, New York, USA). A $P$ value of $<0.05$ was considered to be statistically significant.

\section{Results}

\section{Patients}

A total of 54 patients with MEN1 who underwent screening EUS for PNETs were identified and 38 patients were included in the study ( Fig. 2 ). Patient characteristics, imaging studies performed before the initial EUS, and indication for initial EUS are shown in $>$ Table 1 . Four patients $(11 \%)$ had a history of pancreatic surgery: enucleation in one, distal pancreatectomy in two, and Whipple resection in one. None of the patients had a history of chronic pancreatitis. A total of 169 EUS procedures were included (median 4, IQR 2-5). Median time between procedures was 10.5 months (IQR 6.3-13.0). The majority of included patients underwent EUS every 6-12 months.

\section{PNETS}

In the 54 identified patients, 303 pancreatic lesions were visualized during the screening EUS. In the 38 included patients, a total of 275 focal pancreatic lesions were detected. A total of 226 PNETs were included in the analysis (median 6, IQR 3-8) after excluding completely cystic lesions ( $n=23,8 \%$ ), PNETs detected only once $(n=18,7 \%)$, and PNETs $\geq 20 \mathrm{~mm}$ at the first visualization ( $n=8,3 \%$ ) ( $\triangleright$ Fig. 2). A total of 124 PNETs were visualized during the first EUS procedure ("prevalent" PNETs, $55 \%$ ) in 33 patients ( Fig. 2). During surveillance EUS, an additional 102 incident PNETs were identified (45\%), after a median of 2.4 years (IQR $1.1-3.5$ ) since the initial EUS procedure. A total of 66 PNETs (29\%) were located in the head/uncinate of the pancreas and 160 (71\%) were in the body/tail of the pancreas. At first visualization, 28 PNETs (12.4\%) were $\geq 10 \mathrm{~mm}$ (24 prevalent and 4 incident PNETs). In total, 129 patient follow-up years (median 3.2, IQR 1.5-5.6) and 553 PNET follow-up years (median 2.5, IQR 0.5 - 3.6) were available for analysis.

\section{Size, growth rate, and incidence}

- Table 2 shows size and growth rate of the diameter of the largest axis of all included PNETs, prevalent and incident PNETs, PNETs $<10 \mathrm{~mm}$, and PNETs $\geq 10 \mathrm{~mm}$ at first identification. At the time of first detection, the median size of PNETs $(n=226)$ was $5.0 \mathrm{~mm}$ (IQR 3.7-7.5). Prevalent PNETs were larger at first detection than incident PNETs $(P<0.001)$.

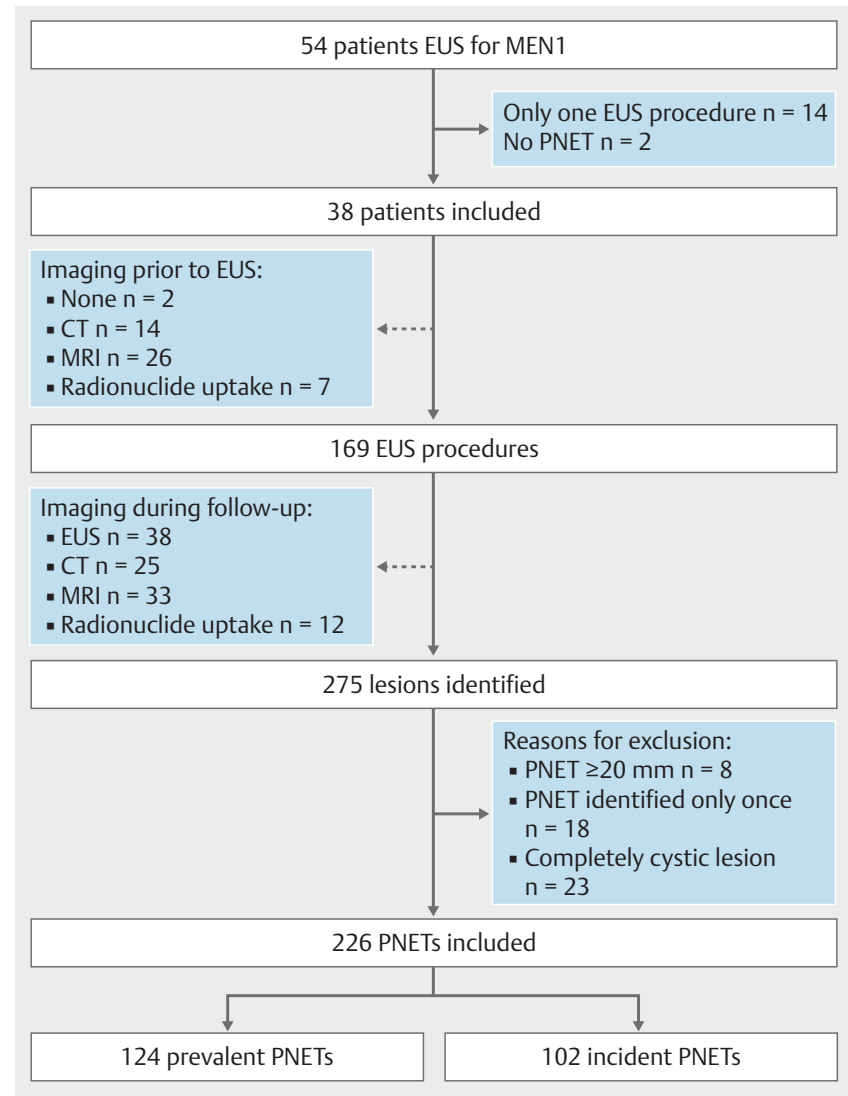

- Fig. 2 Patient inclusion flowchart. EUS, endoscopic ultrasound; MEN1, multiple endocrine neoplasia type 1; PNET, pancreatic neuroendocrine tumor.

The annual growth of the largest diameter of all PNETs ( $n=$ 226) was $0.10 \mathrm{~mm}(P=0.01)$ ( $\triangleright$ Table 2$)$. Prevalent PNETs $(\mathrm{n}=$ 124) grew $0.21 \mathrm{~mm}$ per year $(P<0.001)$ while incident PNETs $(n=102)$ did not grow $(P=0.26)$; this difference was significant $(P<0.01)$. PNETs $<10 \mathrm{~mm}$ at first identification $(n=198)$ did not grow $(P=0.23)$, whereas PNETs $\geq 10 \mathrm{~mm}(n=28)$ grew $0.44 \mathrm{~mm} /$ year $(95 \% \mathrm{Cl} 0.10$ to $0.78 ; P=0.01)$ and this difference was significant $(P<0.001)$. The most rapid growth was seen in prevalent PNETs that were $\geq 10 \mathrm{~mm}$ during the initial EUS $(n=24$; $0.76 \mathrm{~mm}$ per year; $P<0.001)$ ( $>$ Table 2 ).

During follow-up, three PNETs $(3 / 226 ; 1 \%)$ in three patients ( $8 \%$ ) grew to $\geq 20 \mathrm{~mm}, 26-30$ months after the initial EUS. All three PNETs were prevalent PNETs, and sizes at the initial EUS were $14,16.6$, and $18 \mathrm{~mm}$, respectively. In nine patients (24 $\%$ ), the size of 11 PNETs (5\%) increased significantly ( $\geq 5 \mathrm{~mm}$ ), after a median of 2.6 years (IQR 2.3-3.3); all 11 PNETs were prevalent at initial EUS, with a median size of $7.0 \mathrm{~mm}$ (IQR 4.5 10.8). None of the included PNETs metastasized during the study period. Location of the PNET was not correlated with growth $(P=0.87)$. In five patients $(13 \%)$, six PNETs $(3 \%)$ increased two categories in size after a median of 1.0 year (IQR $1.0-1.1)$. The risk of significant growth increased with the number of PNETs visualized in a patient during initial EUS (odds ratio [OR] 1.48 per additional PNET visualized, $95 \% \mathrm{Cl} 1.03$ to $2.11 ; P=$ $0.03)$. 
- Table 1 Baseline characteristics.

\begin{tabular}{|c|c|}
\hline Patient characteristics & $\mathrm{n}=38$ \\
\hline Sex, male, n (\%) & $13(34)$ \\
\hline Age, mean (SD), years & $41(14)$ \\
\hline Surgery prior to EUS, n (\%) & $4(11)$ \\
\hline \multicolumn{2}{|l|}{ Alcohol consumption, $\mathrm{n}(\%)$} \\
\hline None & $12(32)$ \\
\hline $1-2$ unit(s) per week & $19(50)$ \\
\hline 1 unit per day & $3(8)$ \\
\hline 2 units per day & $4(11)$ \\
\hline \multicolumn{2}{|l|}{ Prior imaging modality, n (\%) } \\
\hline None & $2(5)$ \\
\hline CT & $14(37)$ \\
\hline MRI & $26(68)$ \\
\hline Radionuclide uptake scan & $7(18)$ \\
\hline \multicolumn{2}{|l|}{ Indication initial EUS, n (\%) } \\
\hline Doubt about growth & $16(42)$ \\
\hline Suspicion of functional PNET & $14(37)$ \\
\hline Multiple small PNETs & $4(11)$ \\
\hline More detail about location & $3(8)$ \\
\hline Other & $1(3)$ \\
\hline \multicolumn{2}{|l|}{ Imaging during follow up, $\mathrm{n}(\%)$} \\
\hline $\mathrm{CT}$ & $25(66)$ \\
\hline MRI & $33(87)$ \\
\hline Radionuclide uptake scan & $14(37)$ \\
\hline No. of EUS procedures, median (IQR), $\mathrm{n}$ & $4(2-5.3)$ \\
\hline Patient follow-up, median (IQR), years & $3.2(1.1-5.6)$ \\
\hline No. of PNETs, median (IQR), n & $7(4-9)$ \\
\hline PNET characteristics & $n=226(100)$ \\
\hline \multicolumn{2}{|l|}{ Location PNET, n (\%) } \\
\hline Head/uncinate & $66(29)$ \\
\hline Body/tail & $160(71)$ \\
\hline \multicolumn{2}{|l|}{ Size PNET, mm } \\
\hline $0-4$ & $99(44)$ \\
\hline $5-9$ & $99(44)$ \\
\hline $10-14$ & $21(9)$ \\
\hline $15-19$ & $7(3)$ \\
\hline \multicolumn{2}{|l|}{ PNET follow-up, median (IQR), years } \\
\hline Total & $2.5(0.5-3.6)$ \\
\hline Prevalent PNETs & $2.6(0.6-3.6)$ \\
\hline Incident PNETs & $2.4(0.0-3.6)$ \\
\hline
\end{tabular}

The annual detection rate of new PNETs (incidence rate) was 0.79 per patient-year of follow-up ( $95 \% \mathrm{Cl} 0.73$ to 0.87 ). One incident PNET was $20 \mathrm{~mm}$ at first visualization, during EUS performed just 1.5 months after the initial EUS procedure; none of the remaining incident PNETs grew to $\geq 15 \mathrm{~mm}$ during followup.

No differences were seen between the operator who performed $78 \%$ of the procedures and the remaining three operators with regard to number of PNETs visualized during each EUS ( $P=$ 0.65 after correction for timing of EUS), incident PNET detection rate $(0.85$ vs. $0.55 ; P=0.08)$, or size of incident PNETs $(P=$ 0.57 after correction for timing of EUS).

\section{Surgery}

During follow-up, 12 surgical resections were performed in 11 patients (29\%) ( $\triangleright$ Table 3 ). In five patients, surgery was performed for PNETs that were $\geq 20 \mathrm{~mm}$ at first detection. Seven patients underwent surgery after surveillance EUS for PNETs that were $<20 \mathrm{~mm}$ at first detection; this was done because of growth of the PNET $(n=4)$ or because the PNETs were hormone-producing and conservative treatment was unsatisfactory $(n=3)$. All PNETS $\geq 10 \mathrm{~mm}$ that prompted surgical resection had also been visualized with CT/MRI less than 1 year before surgery. In one patient, the PNETs were only visualized with EUS prior to surgery; in this patient, however, elevated chromogranin A levels were detected.

\section{Discussion}

This EUS-based surveillance study shows that the growth of small PNETs $(<20 \mathrm{~mm})$ in a large cohort of patients with MEN1 is slow. Only a few small PNETs exceeded $20 \mathrm{~mm}$ during followup. The risk of significant growth was higher if a PNET was $\geq 10$ $\mathrm{mm}$ and increased if a higher number of PNETs was found. The incidence rate of new PNETs was high, but these PNETs were often $<10 \mathrm{~mm}$ and did not grow during follow-up. One incident PNET was $20 \mathrm{~mm}$ at discovery and required surgery, but none of the remaining 101 incident PNETs exceeded $15 \mathrm{~mm}$ during follow-up, suggesting that clinical relevance of incident PNETs is limited. Finally, all PNETs $\geq 10 \mathrm{~mm}$ for which surgery was performed were also detected with CT/MRI.

Our findings on growth rate of small, asymptomatic PNETs in patients with MEN1 are in line with the results of a recent, nationwide study in The Netherlands [25]. In this study, CT and MRI were used to assess growth rate of PNETs $(n=92)$ over a median follow-up period of 4 years (range $0.5-16.5$ ). Median baseline tumor size in the study $(12.0 \mathrm{~mm}$, range $3-82)$ was larger than in our study, which is probably due to the fact that CT and MRI are less suitable for detecting lesions $<10 \mathrm{~mm}$ [4]. Even though much larger PNETs were included, the authors reported no clinically relevant growth ( $<1 \mathrm{~mm}$ per year). Sakurai et al. [26] reported on 13 prevalent PNETs using CT with a follow-up ranging from 24 to 115 months. Only one PNET showed significant growth (>20\%), but this PNET was already $31 \mathrm{~mm}$ during the initial EUS. In another study, the Groupe des Tumeurs Endocrines reported an increase of PNET size of at least two categories (median baseline size $6.0 \mathrm{~mm}$, range $2-60$ ) in 2 of 16 patients 
- Table2 Size and annual growth of pancreatic neuroendocrine tumors.

\begin{tabular}{|c|c|c|c|c|}
\hline & $\mathbf{n}$ & Initial EUS, median (IQR), mm & Annual growth (95\%Cl), mm & $P$ value \\
\hline PNETS & 226 & $5.0(3.7-7.5)$ & $0.10(0.02$ to 0.19$)$ & 0.01 \\
\hline \multicolumn{5}{|l|}{ Prevalent } \\
\hline$<10 \mathrm{~mm}$ & 100 & $5.0(4.0-7.1)$ & $0.11(0.003$ to 0.21$)$ & 0.04 \\
\hline$\geq 10 \mathrm{~mm}$ & 24 & $12.0(10.8-15.0)$ & $0.76(0.42$ to 1.11$)$ & $<0.001$ \\
\hline Total & 124 & $6.0(4.0-8.5)$ & 0.21 (0.10 to 0.32 ) & $<0.001$ \\
\hline \multicolumn{5}{|l|}{ Incident } \\
\hline$<10 \mathrm{~mm}$ & 98 & $4.2(3.0-5.9)$ & $-0.03(-0.13$ to 0.07$)$ & 0.54 \\
\hline$\geq 10 \mathrm{~mm}$ & 4 & $12.3(11.3-12.7)$ & $-0.63(-1.47$ to 0.21$)$ & 0.17 \\
\hline Total & 102 & $4.4(3.0-6.0)$ & $-0.06(-0.18$ to 0.05$)$ & 0.26 \\
\hline
\end{tabular}

after a median follow-up of 50 months (range 12 - 70) with EUS, resulting in a patient number-needed-to-test of 8 , comparable to the number we found (5 of 38 patients; 7.6) [24]. In another EUS-based surveillance study by Kann et al., median baseline size of PNETs was also $5 \mathrm{~mm}$ [17]. However, the authors reported a growth of $1.3 \%$ (SD $3.2 \%$ ) per month, which would result in an annual growth of $16.8 \%$ per year ( $1 \mathrm{~mm}$ in the first year), whereas we found an annual growth of $0.10 \mathrm{~mm}(2 \%)$. Fewer patients $(n=20)$ and fewer PNETs $(n=61)$ were included in that study. Moreover, considerably fewer patient and tumor followup years were available than in the current study: 33.8 vs. 129 patient-years and 106.7 vs. 553 tumor-years, respectively. Furthermore, only four patients (11\%) from our cohort had a history of pancreatic surgery, whereas 10 patients (50\%) in the study by Kann et al. had previously undergone surgery [17]. These findings suggest that patients referred to Kann's institution had a more aggressive disease course.

We found that prevalent PNETs grew significantly faster than incident PNETs $(P<0.01)$, unlike a recent study in which incident PNETs were found to grow significantly faster than prevalent PNETs $(P=0.01)$ [18]. Although the study by D'souza et al. had a long follow-up, only 11 patients with 30 PNETs (18 prevalent PNETs and 12 incident PNETs) were included. Furthermore, EUS data from 1990-2010 were used and cystic lesions were included. Although age at the initial EUS was comparable (41 vs. 42 years), we found more (3.3 vs. 1.6 PNETs/patient) but smaller PNETs $(6.0 \mathrm{~mm}$ vs. $9.5 \mathrm{~mm})$ during initial EUS. This suggests that in the other study, small prevalent PNETs may have been missed initially and were only visualized after having grown larger, thus erroneously being considered an incident PNET. Moreover, 8 of 11 patients had a history of distal pancreatectomy and $9 / 18$ prevalent PNETs (50\%) were $\geq 10 \mathrm{~mm}$ during initial EUS; in our cohort this was seen in only 24/124 (19\%) PNETs. Finally, technical advancements in EUS equipment in our more recent cohort may have played an important role as small PNETs are easily missed; this is demonstrated by the fact that often more PNETs are found during pancreatectomy than during imaging studies [15, $20-23,27]$.
The incidence rate of new PNETs reported by Kann et al. was comparable to our findings: 0.62 vs. 0.79 PNETs per patientyear follow-up, respectively [17]. D'souza et al. reported 12 incidental lesions during 72.4 patient-years of follow-up, resulting in an incidence rate of just 0.17 PNETs per patient-year [18]. Sakurai et al. reported 6 new lesions during 91 follow-up years, resulting in an incidence of just 0.07 PNETs per patientyear [26]. The lower incidence rate of this latter study is explained by the fact that CT was used to detect PNETs. It is likely that lesions that were considered incident PNETs in the study by Sakurai et al. were in fact already prevalent during an earlier imaging study, but were too small to be detected.

The current study suggests that the clinical benefit of surveillance EUS may be limited. Previous studies have shown that a size $\geq 20 \mathrm{~mm}$ is a predictive and easily workable cutoff point to identify patients at risk for PNET-related metastasis [12, 15, 24, $28,29]$. The decision to perform surgery is often based on the largest PNET, thus limiting the clinical relevance of detecting smaller PNETs. As can be seen in $>$ Table 3 , surgically resected PNETs $\geq 10 \mathrm{~mm}$ were all visualized with CT/MRI beforehand. Furthermore, EUS surveillance may not be useful if no PNETs are visualized during the initial EUS, since incident PNETs almost never grow beyond $20 \mathrm{~mm}$. One "incident" PNET in our study was $20 \mathrm{~mm}$ when it was first mentioned in the endoscopy report, and it was described as having a lymph node-like appearance. This EUS procedure was performed only 1.5 months after the initial EUS procedure. There was no mention of the PNET in the initial EUS report, while the PNET was already visible on CT/ $M R I$, and EUS was repeated to visualize the lesion to make an informed decision about surgical intervention. During surgery, the lesion was found in the tail of the pancreas and the tail was resected. It is likely that the lesion was prevalent during the initial EUS, but that there was no mention of it in the report because it was considered a lymph node.

Initial screening and surveillance for PNETs in patients with MEN1 can be performed with CT/MRI, as lesions $\geq 10 \mathrm{~mm}$ are found at least as often with MRI as with EUS, and asymptomatic PNETs $<10 \mathrm{~mm}$ have limited clinical relevance [19]. If a patients' symptomatology suggests a functional PNET, then EUS is the 


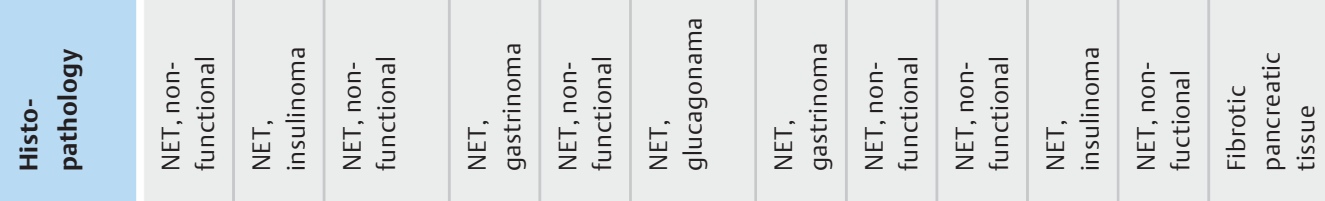

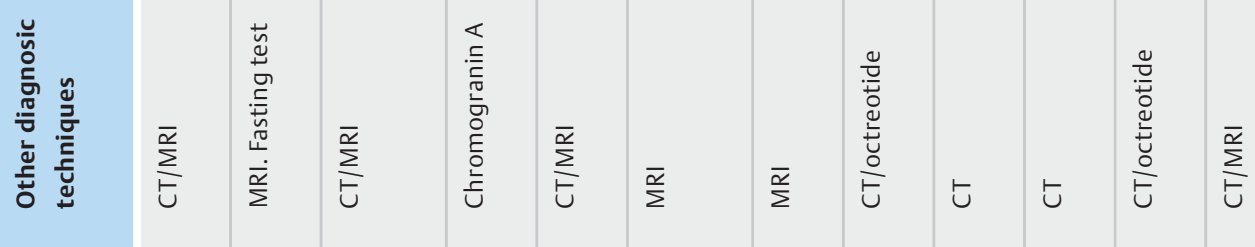

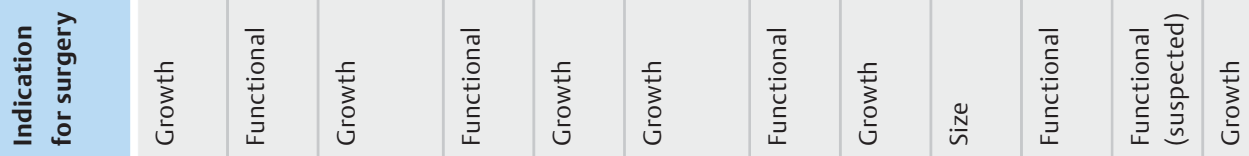

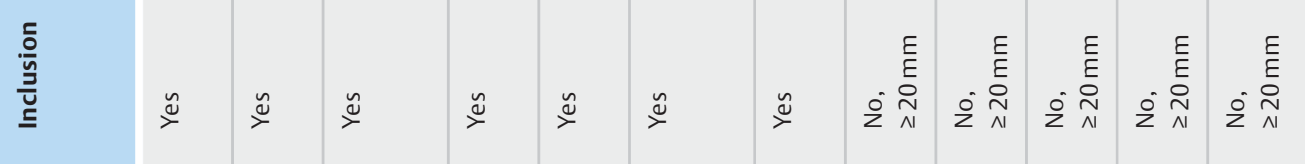

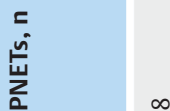

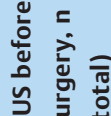

岂产产

Ð

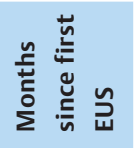

ตे i $\hat{m}$

妾

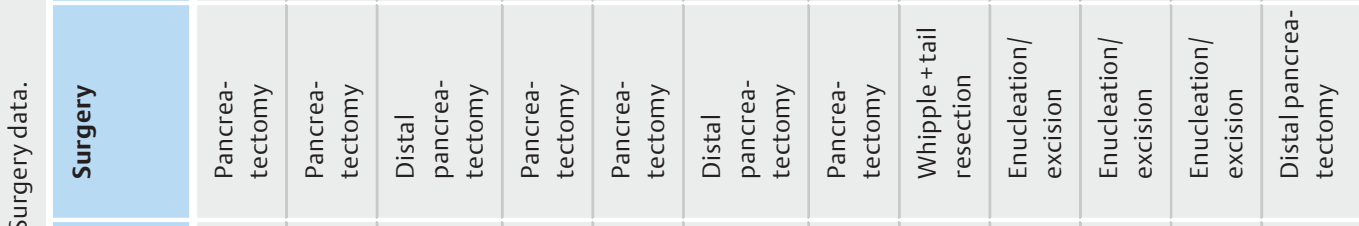




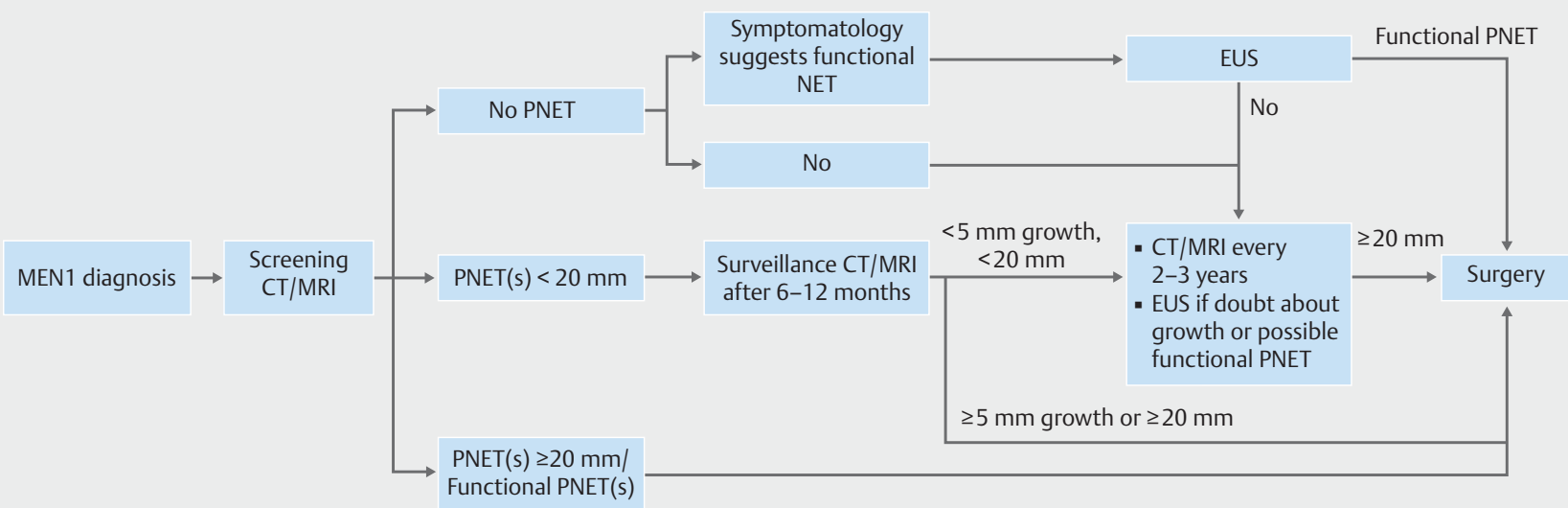

- Fig. 3 Proposed screening and surveillance imaging strategies for pancreatic neuroendocrine tumors in multiple endocrine neoplasia type 1 (MEN1). CT, computed tomography; MRI, magnetic resonance imaging; PNET, pancreatic neuroendocrine tumor; EUS, endoscopic ultrasound.

imaging modality of choice if CT/MRI shows no PNET, as EUS is the most sensitive modality for detecting smaller PNETs $[4,9,10,19,20]$. Based on the findings of the current study, if a PNET of $10-19 \mathrm{~mm}$ is identified during screening CT/MRI, then surveillance imaging should be performed after $6-12$ months to avoid potential delay in surgery. If only PNETs $<10 \mathrm{~mm}$ are visualized during screening, surveillance CT/MRI after 6-12 months can be useful to assess whether there is growth of the PNETs. If limited growth of the PNETs (i.e. $<5 \mathrm{~mm}$ ) is observed at that time and no PNET is $\geq 20 \mathrm{~mm}$, periodic surveillance imaging using MRI or CT with longer intervals (e.g. once every $2-3$ years) may be justified. Additional EUS imaging can then be performed when symptomatology suggests a functional NET but $C T / M R I$ show no PNET, or when there is doubt about growth of a PNET on CT/MRI ( Fig. 3). This approach is largely in line with previous recommendations [15].

This study has some potential limitations. Histology assessment was not available in the majority of PNETs. However, obtaining histology of pancreatic lesions in MEN1 is not common in clinical practice or clinical studies, as the a priori chance that a pancreatic lesion is a PNET is very high in patients with MEN1 [4, 17-23]. In certain cases, histological assessment could be considered when there is doubt about neuroendocrine origin of the lesion (i.e. when EUS features are suggestive of adenocarcinoma). However, we never encountered such a dilemma. Dedicated effort went into allocating measurements to the correct PNET by using a hierarchal model. Nevertheless, measurements within one region of the pancreas were grouped based on size if no further description of the PNETs was available. Theoretically, it is possible that an incident PNET grew larger than a previously visualized PNET between two EUS procedures. However, no growth was observed in solitary incidental PNETs, in which re-identification is not difficult. Although this shortcoming may be more profound in a retrospective study, a prospective study would require similar assumptions. Unless a PNET has distinct features, allocating measurements to the correct PNET can only be done based on the previously reported location and size. It is seldom possible to determine the exact location of a PNET on previously recorded images. Therefore, the ultrasonographer needs to rely largely on the previous EUS report; this is a well-known shortcoming of (E)US.

This was a single-center study, which can be considered either an advantage or a disadvantage. The fact that one experienced ultrasonographer performed $78 \%$ of the EUS procedures reduces operator bias; on the other hand, it limits generalizability of the study outcomes. However, no differences were observed between the operators with regard to number of lesions found or incident PNET detection rate. As MEN1 is rare and care for these patients requires a multidisciplinary approach with experienced physicians, treatment is centralized in The Netherlands. Therefore, limited generalizability is not a clinically relevant shortcoming in the context of care for patients with MEN1.

In conclusion, based on this large cohort, small PNETs in patients with MEN1 grow more slowly than previously thought, and EUS-based surveillance of these PNETs appears to have limited clinical value. Although PNETs increase in size, few patients had a PNET that grew to $\geq 20 \mathrm{~mm}$. Only prevalent PNETs with a size of $\geq 10 \mathrm{~mm}$ at initial visualization have a relatively high risk of showing significant growth; PNETs $<10 \mathrm{~mm}$ and incident PNETs did not grow. Therefore, the interval between surveillance imaging could probably be prolonged if only small, asymptomatic PNETs are found during the initial screening. Furthermore, standard EUS surveillance does not appear warranted because of the limited additional value when compared with noninvasive imaging modalities.

\section{Competing interests}

\section{None}




\section{References}

[1] Sadowski SM, Triponez F. Management of pancreatic neuroendocrine tumors in patients with MEN 1. Gland Surgery 2015; 4: 63-68

[2] Goudet P, Murat A, Binquet $C$ et al. Risk factors and causes of death in MEN1 disease. A GTE (Groupe d'Etude des Tumeurs Endocrines) cohort study among 758 patients. World J Surg 2010; 34: 249-255

[3] Walls GV. Multiple endocrine neoplasia (MEN) syndromes. Semin Pediatr Surg 2014; 23: 96 - 101

[4] van Asselt S], Brouwers AH, van Dullemen HM et al. EUS is superior for detection of pancreatic lesions compared with standard imaging in patients with multiple endocrine neoplasia type 1. Gastrointest Endosc 2015; 81: 159-167

[5] Metz DC, Jensen RT. Gastrointestinal neuroendocrine tumors: pancreatic endocrine tumors. Gastroenterology 2008; 135: 1469-1492

[6] Pieterman CR, Conemans EB, Dreijerink KM et al. Thoracic and duodenopancreatic neuroendocrine tumors in multiple endocrine neoplasia type 1: natural history and function of menin in tumorigenesis. Endocr Relat Cancer 2014; 21: R121-142

[7] Dean PG, van Heerden JA, Farley DR et al. Are patients with multiple endocrine neoplasia type I prone to premature death? World J Surg 2000; $24: 1437-1441$

[8] Pieterman CR, Schreinemakers JM, Koppeschaar HP et al. Multiple endocrine neoplasia type 1 (MEN1): its manifestations and effect of genetic screening on clinical outcome. Clin Endocrinol 2009; 70: 575581

[9] Hellman P, Hennings J, Akerstrom G et al. Endoscopic ultrasonography for evaluation of pancreatic tumours in multiple endocrine neoplasia type 1 . Br J Surg 2005; 92: 1508-1512

[10] Lewis MA, Thompson GB, Young WFJr. Preoperative assessment of the pancreas in multiple endocrine neoplasia type 1. World J Surg 2012; 36: $1375-1381$

[11] Thakker RV, Newey PJ, Walls GV et al. Clinical practice guidelines for multiple endocrine neoplasia type 1 (MEN1). J Clin Endocrinol Metab 2012; 97: 2990 - 3011

[12] Bettini R, Partelli S, Boninsegna L et al. Tumor size correlates with malignancy in nonfunctioning pancreatic endocrine tumor. Surgery 2011; 150: 75-82

[13] Falconi M, Bartsch DK, Eriksson B et al. ENETS Consensus Guidelines for the management of patients with digestive neuroendocrine neoplasms of the digestive system: well-differentiated pancreatic nonfunctioning tumors. Neuroendocrinology 2012; 95: 120-134

[14] Kulke MH, Anthony LB, Bushnell DL et al. NANETS treatment guidelines: well-differentiated neuroendocrine tumors of the stomach and pancreas. Pancreas 2010; 39: 735-752

[15] Triponez F, Goudet P, Dosseh D et al. Is surgery beneficial for MEN1 patients with small $(<\mathrm{or}=2 \mathrm{~cm})$, nonfunctioning pancreaticoduodenal endocrine tumor? An analysis of 65 patients from the GTE World J Surg 2006; 30: 654-662

[16] Kann PH, Kann B, Fassbender WJ et al. Small neuroendocrine pancreatic tumors in multiple endocrine neoplasia type 1 (MEN1): least significant change of tumor diameter as determined by endoscopic ul- trasound (EUS) imaging. Exp Clin Endocrinol Diabetes 2006; 114: $361-365$

[17] Kann PH, Balakina E, Ivan D et al. Natural course of small, asymptomatic neuroendocrine pancreatic tumours in multiple endocrine neoplasia type 1: an endoscopic ultrasound imaging study. Endocr Relat Cancer 2006; 13: 1195-1202

[18] D'souza SL, Elmunzer BJ, Scheiman JM. Long-term follow-up of asymptomatic pancreatic neuroendocrine tumors in multiple endocrine neoplasia type I syndrome. J Clin Gastroenterol 2014; 48: 458 461

[19] Barbe C, Murat A, Dupas B et al. Magnetic resonance imaging versus endoscopic ultrasonography for the detection of pancreatic tumours in multiple endocrine neoplasia type 1. Dig Liver Dis 2012; 44: 228 234

[20] Langer P, Kann PH, Fendrich $\vee$ et al. Prospective evaluation of imaging procedures for the detection of pancreaticoduodenal endocrine tumors in patients with multiple endocrine neoplasia type 1 . World ] Surg 2004; 28: $1317-1322$

[21] Gauger PG, Scheiman JM, Wamsteker EJ et al. Role of endoscopic ultrasonography in screening and treatment of pancreatic endocrine tumours in asymptomatic patients with multiple endocrine neoplasia type 1. Br J Surg 2003; 90: 748-754

[22] Anlauf M, Schlenger R, Perren A et al. Microadenomatosis of the endocrine pancreas in patients with and without the multiple endocrine neoplasia type 1 syndrome. Am J Surg Pathol 2006; 30: 560 - 574

[23] Gauger PG, Doherty GM, Broome JT et al. Completion pancreatectomy and duodenectomy for recurrent MEN-1 pancreaticoduodenal endocrine neoplasms. Surgery 2009; 146: $801-808$

[24] Thomas-Marques L, Murat A, Delemer B et al. Prospective endoscopic ultrasonographic evaluation of the frequency of nonfunctioning pancreaticoduodenal endocrine tumors in patients with multiple endocrine neoplasia type 1. Am J Gastroenterol 2006; 101: 266-273

[25] Pieterman CR, de Laat JM, Twisk JW et al. Growth rate of MEN1-related pancreatic neuroendocrine tumors: new insights based on the results from the DutchMEN1 Study Group (DMSG) National MEN1 Database 1990-2010. Abstract presented at Endocrine Society's 97th Annual Meeting and Expo; 2015 March 5-8; San Diego, California. Endocrine Reviews 2015; 36: 2-3 Available from http://press.endocrine.org/ doi/abs/10.1210/endo-meetings.2015.TB.2.PP02-3 Accessed: 12 May 2016

[26] Sakurai A, Katai M, Yamashita K et al. Long-term follow-up of patients with multiple endocrine neoplasia type 1. Endocr J 2007; 54: 295 302

[27] Skogseid B. Multiple endocrine neoplasia type 1. Br ] Surg 2003; 90: $383-385$

[28] Toste PA, Kadera BE, Tatishchev SF et al. Nonfunctional pancreatic neuroendocrine tumors $<2 \mathrm{~cm}$ on preoperative imaging are associated with a low incidence of nodal metastasis and an excellent overall survival. J Gastrointest Surg 2013; 17: 2105-2113

[29] Haynes AB, Deshpande V, Ingkakul T et al. Implications of incidentally discovered, nonfunctioning pancreatic endocrine tumors: short-term and long-term patient outcomes. Arch Surg 2011; 146: 534- 538 